\title{
Impact of Drone Technology in Agriculture
}

\author{
Vijay Rana ${ }^{1 *}$ and Mahima ${ }^{2}$ \\ ${ }^{1}$ Sant Baba Bhag Singh University, Jalandhar, India \\ ${ }^{2}$ Gurugram University, Noida, India \\ *Corresponding author
}

Keywords

Unmanned Aerial

Vehicles (UAVs),

Drones, Artificial

Intelligent

Article Info

Accepted:

15 December 2019

Available Online:

20 January 2020

\section{A B S T R A C T}

This work defines the benefits of drone technology in agriculture, and their application areas, describing from examples how drones function on fields and also highlights the different features of drone for managing the crops by applying precise cameras to identify pests and water shortages. For agricultural applications, summarized smart/intelligent farming techniques are being utilized such as exercise of unmanned aerial vehicles (UAVs). The UAVs technique is combination of both communication and information technologies, robots, artificial intelligence, big data, and the Internet of Things. The article concludes by recommending that more farmers work with drone technique to improved their agricultural results. In this, we consider the recent trends of research to employ agricultural UAVs, control technologies, equipment, and development.

\section{Introduction}

Unmanned Aerial Vehicles (UAVs) is known as Drones that are regarded as pilotless aircraft techniques utilized in various areas such as Industrial monitoring system, shooting, battleground observation, space ambulance, product liberation and several more applications. It is controlled by specific point and useful as diminutive distance flying areas. In agriculture, there are various functions of drone tools like crop supervising, crop quantity and verve considerations, crop record, production of recommendation records, meticulousness drenching, and examination of farm infrastructure, high declaration mapping and examination of individual areas, crop scratch measurement \& insurance claim forensics. Drones recommend high eminence imaging, which can assist observe crops while scanning and evaluating areas to accumulate essential agricultural information, This imaging technique can help in the determination of crops and their growth such as their health, and the identification of their promptness, these images can gives farmers with the capability to identify how grown their crops are, and if and when they 
will be prepared for harvest. This Imaging technique can help with overall field organization, giving results in real-time determining where precise crops may need much water, fertilizer, soil or pesticides. Agricultural drones allow users/farmers to watch their fields from the sky and depiction intending problems on the farm such as irrigation issues, soil dissimilarity as well as pest and fungal infestations, having determined these issues, the farmer can come up with better results to develop crop supervision and production. The current research shown that almost $85 \%$ of drone machinery is mostly used by military and rest $15 \%$ used by civilians for various applications.

However, with the rapid growth of technology, it is reported that growth of Drones technology is increasing 25-32\% every year especially in the area of Agriculture. Lots of researchers and drones manufacturing companies are either coming up or in process of releasing varied models of Drones especially made up for agriculture.

\section{Impact of drones technology in agricultural}

Drone technique provides a high technologies makeover to agriculture industry with planning and stratagem based on real-time applications for collecting and progression. It is assumed that Drones marketplace will reach $\$ 200$ billion by year 2023. There are many applications, where drones can play an important role throughout the crop cycle:

\section{Soil and pasture examination}

Drone technology is very reliable technique by farmers to examine the farm situation at the starting of every crop year. Drones produce 3-D maps images for soil examination that is functional for farmers to take care during seed plugging. Soil and pasture investigation via drones also gives beneficial information for irrigation and organization nitrogen level of fields for improved crop expansion.

\section{Planting}

Recently research states that drone is able to plant trees on a massive scale. The drone works by flying across a particular region, gathering information about soil conditions and identifying the prime locations for planting. Five drones, operated by two operators, can plant 200,000 trees a day. According to the survey of World Economic Forum [WEF], the earth cuts down approximately 16 billion trees but only plants 10 billion, because re-fixing physically is very deliberate and costly. The drone-based technology can help to plant trees 10 times quicker than humans, for $8 \%$ less cost, and in places humans can't reach. It fires nutrient pods which contain a seed into the soil, at just the right depth. Initial results suggest it could decrease planting costs by as much as $85 \%$.

Drones technology shoot pods with seeds and plant nutrients into the soil, presenting the plant each the nutrients essential to maintain life.

\section{Monitoring Crops}

The drone technology is used for monitoring crops and finds damage to plants after a storm, appraise crop growth and determine areas that are being below fertilizing and irrigated. It provides $2 \mathrm{D}$ and $3 \mathrm{D}$ images and permitting farmers to measure plant growth, crop numbers and thickness.

Evaluating the 2D/3D images can gives valuable results on the expansion of crops and reveals fabrication inefficiencies. Drone works with sensors system to monitor and sense reflected Near Infra-Red (NIR) light. 
NIR is valuable because strong plants absorb perceptible light and reflect NIR, while less strong plants absorb more NIR and reflect more visible light. NIR measure provides maps information of plant health to farmers and supervises remedies more specifically. Drone crop monitoring facilities real time crop vegetation index monitoring via spectral examination of high declaration satellite images for dissimilar areas and crops that permits to monitor optimistic and pessimistic dynamics of crop growth.

\section{Health Evaluation of Plants}

Syndrome can unconstructively impact plant health before some noticeable signs like leaf discolouration functions. While these functions are imperceptible to the naked eye, cameras and utilizing particular filters could notice these subtle changes. The drone techniques facilitate to examine plant health and mark crop inconsistency in minutes.

By utilizing plant health algorithms such as NDVI with drone maps can assist emphasize inconsistency and take it easiest to spot crop stress, but there's a several of perplexity out there about NDVI. It's necessary to evaluate crop health and spot bacterial or fungal infections on plants. By evaluating a crop utilizing equally visible and near-infrared light, drone-carried system can recognize that plants reflect dissimilar quantities of green light and NIR light. This data produced multispectral images that summarized changes in plants and specify their health.

\section{Role of Artificial Intelligence in Agriculture}

Artificial intelligence plays an important role in agriculture, because agriculture is a main part of the base of our economy, as environments are changing \& populations are increasing, Artificial Intelligence is becoming a scientific modernism that is enhancing \& protecting crop yield. The basic applications of Artificial Intelligence technology in agriculture fields are divided into three major forms that are Agricultural Robots, Predictive Analytics, Crop \& Soil Monitoring. The machine learning algorithms are utilized to progression information fetched by drones to observe crop \& soil health. Some major artificial intelligence techniques are:

\section{Agricultural Robots}

Agricultural Robot is utilized to hold vital agricultural jobs likes as harvesting crops at a superior level \& earlier rapidity than human laborers, Robots are formalized to help in picking \& packing crops while contesting other issues within the agricultural labor work, Agricultural robots may keep crops from injurious tidies which can be defiant to herbicide elements, which are meant to eradicate them.

The agriculture robot may harvest 10 acres in a one day and replace 25 human laborers, An approximation $38 \%$ of yearly field costs are funneled into "wages, salaries and contract labor expenditures" in the areas of crops likes fruits and vegetables where labor requires tend to be the highest. It also helps to human workers from the injurious results of tackling the chemicals by the hand and during the system of high spraying, and they can decrease up to $75 \%$ of a farms utilize of pesticides.

\section{Plantix app}

Plantix app determines the possible imperfections \& nutrient deficiencies in soil. This app utilizes pictures to identify plant diseases with image recognition work to determine probable defects through images fetched by the Smartphone cameralhigh resolution cameras. The farmers can 
contribute in the online users to app users network to converse plant health problems and access their location weather reports.

\section{Driverless tractors}

A driverless tractor is an autonomous farm vehicle that works without driver with high tractive attempt at measured speeds for the intention of agricultural tasks. It presents a safer, less stressful working environment for farm workers and their families. It designed to examine their arrangement, choose the pace and avoid barriers like animals, human beings or objects in the field performing their task. It works with the help of a administrator monitoring the evolution at a control position with remote control from a expanse.

Figure.1 Agriculture Field (Left) and Fixed Wing Drone (Right)

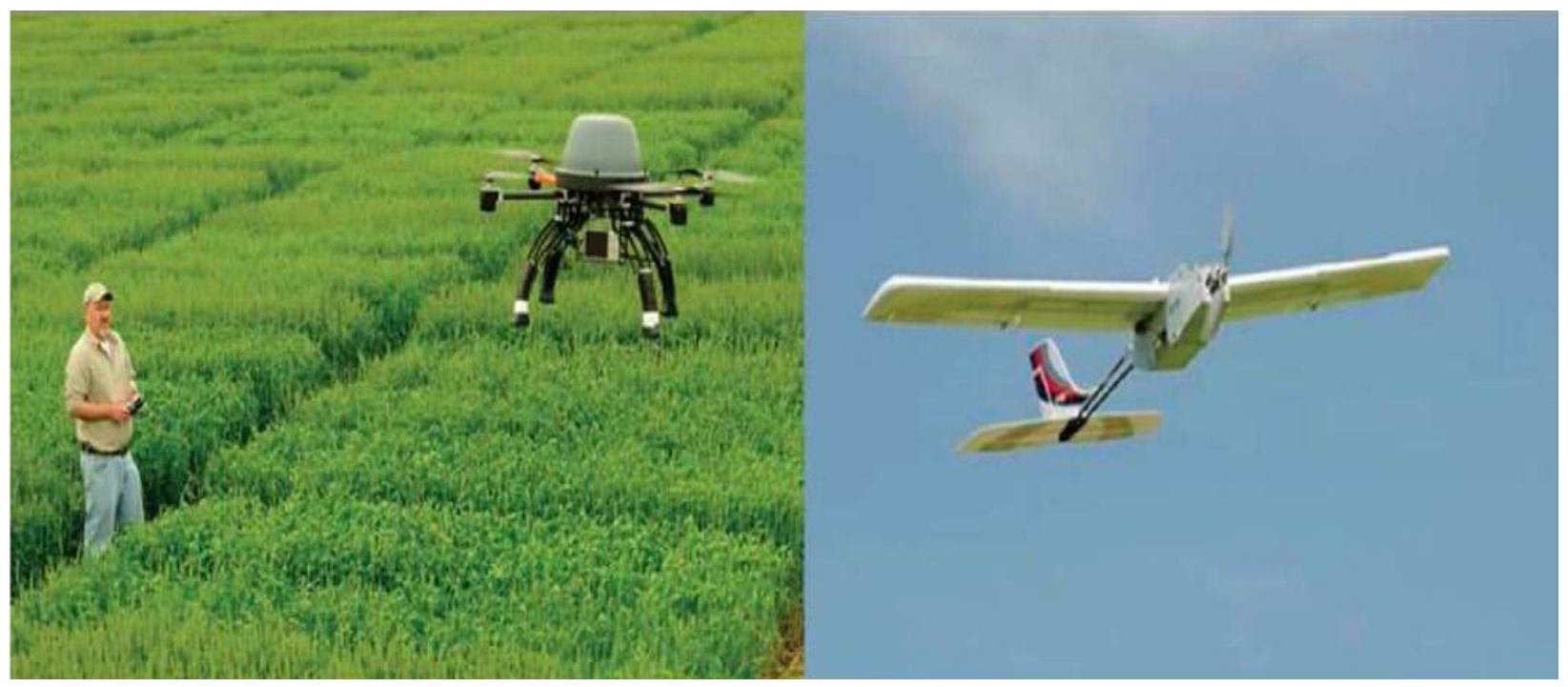

Figure.2 Analyzing Soil and Fields

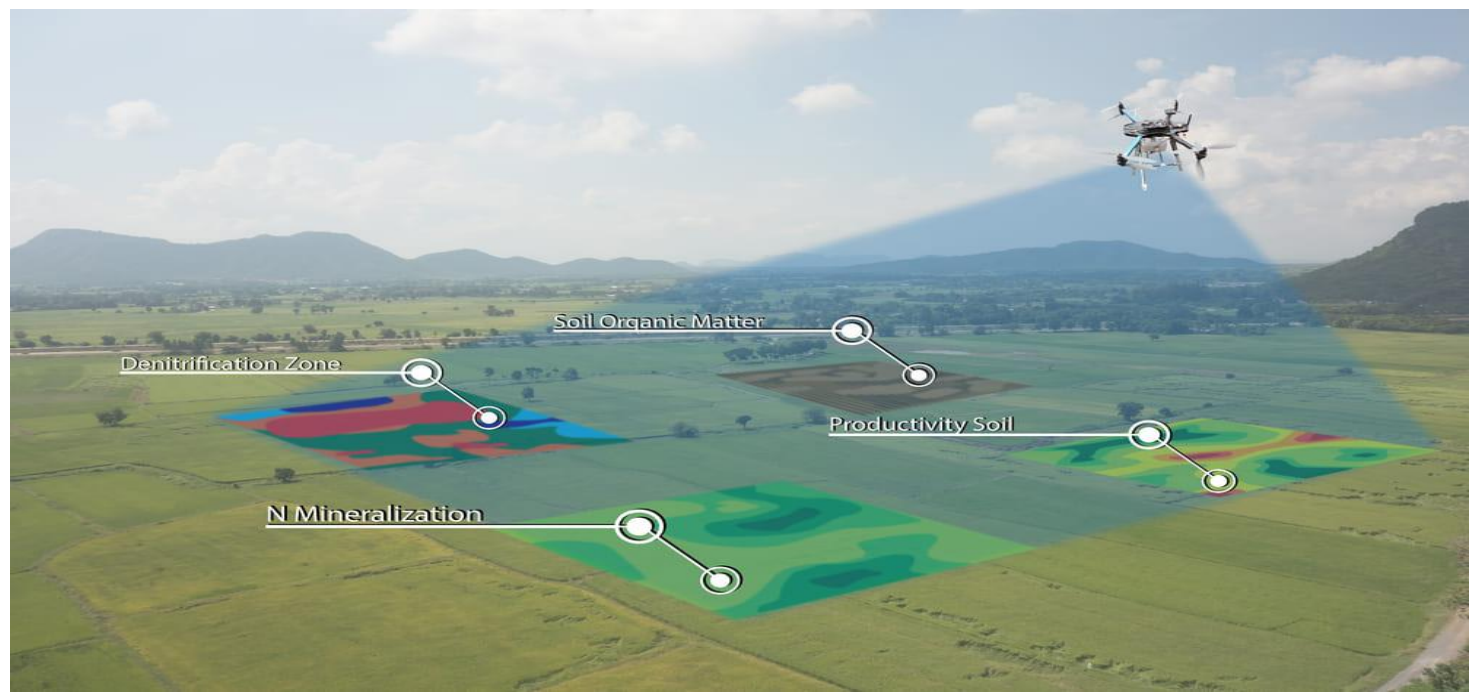


Figure.3 Tree Plantation with Drones

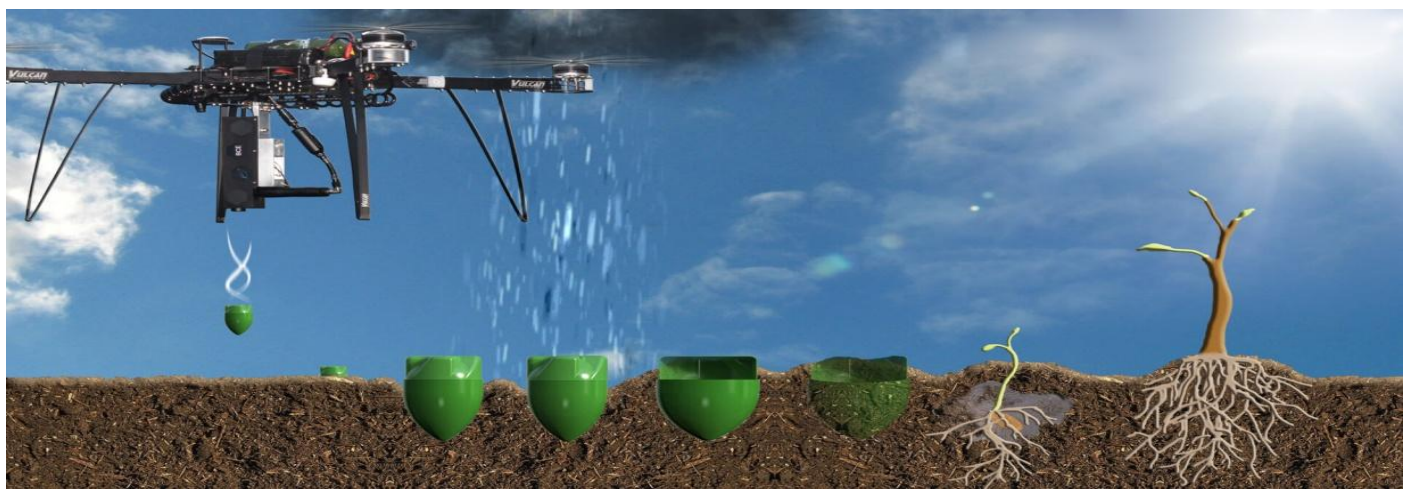

Figure.4 Crops Monitoring Measures

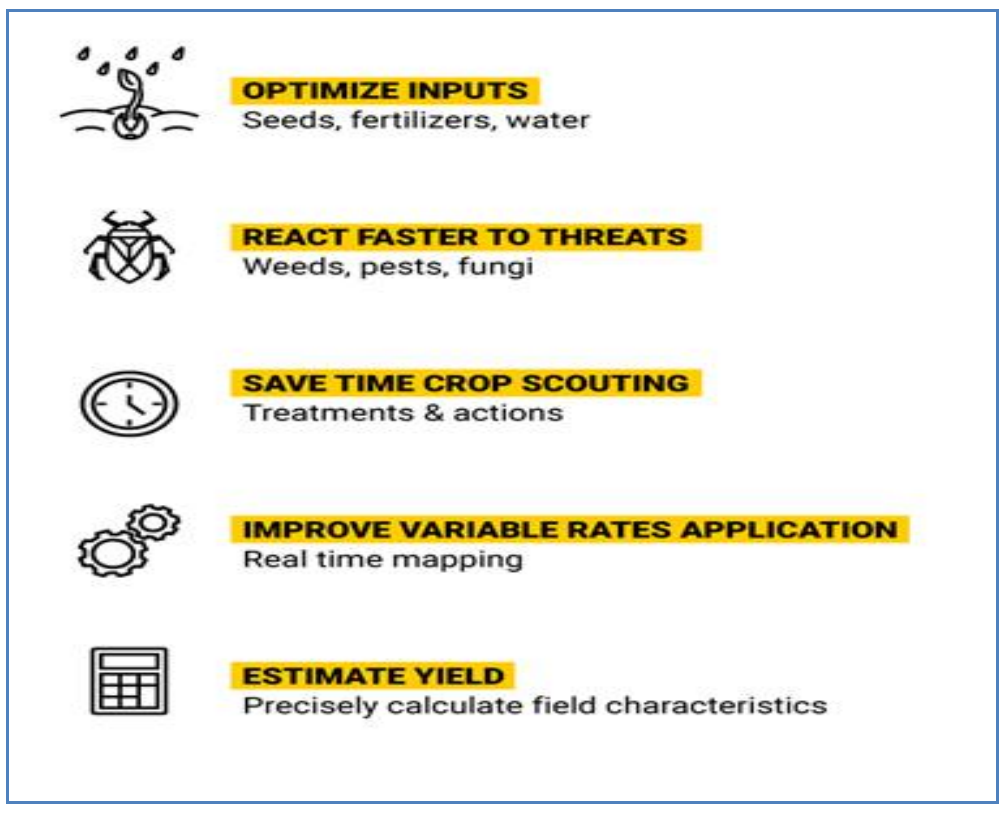

Figure.5 Plant Health Monitoring

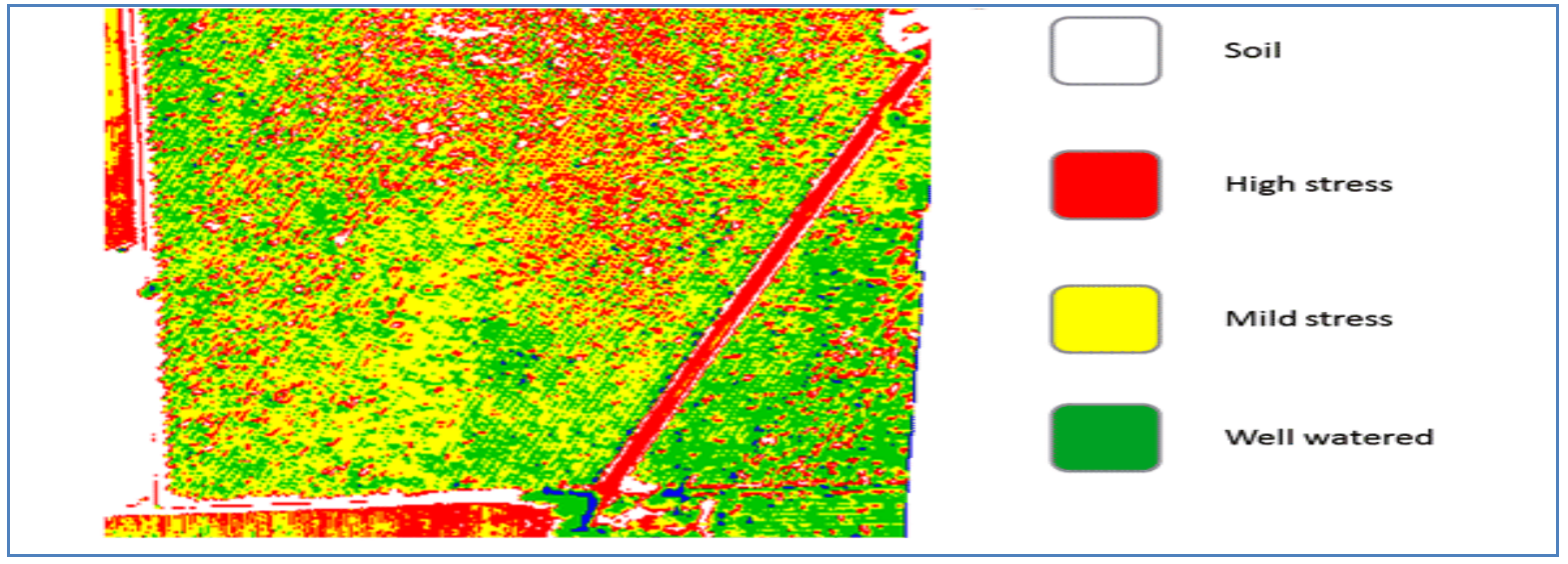


Figure.6 Agriculture Robotics

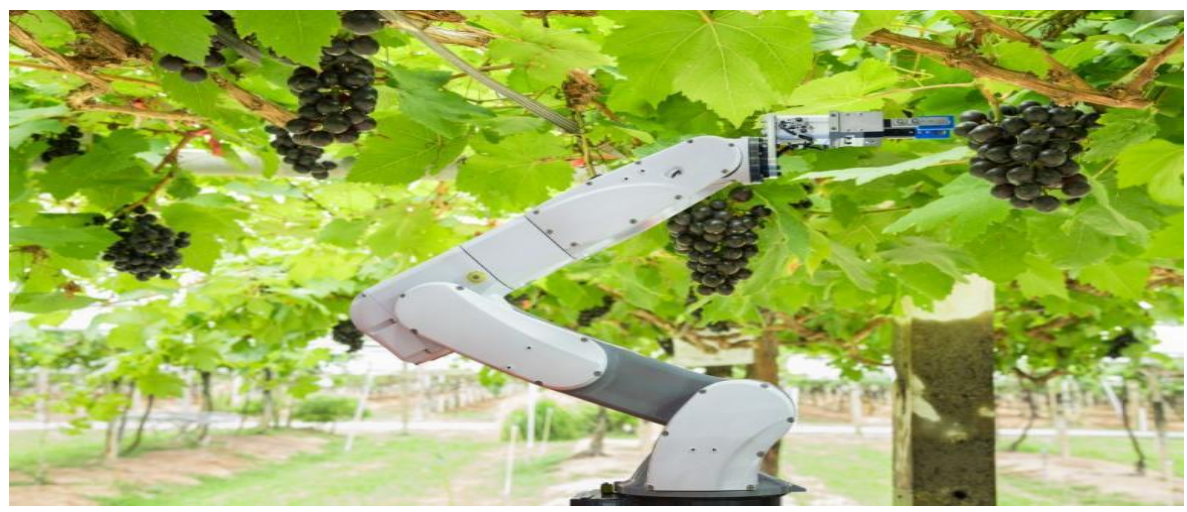

\section{Precise Farming}

Recent research described that exactitude farming utilized Artificial intelligence to make precise and prohibited methods that assist present direction \& appreciative about water and nutrient executive, most favorable harvesting and planting. These techniques formulate farming more proficient, and can assist forecast ROI on precise crops depend on their costs and periphery within the market.

In conclusion, the review results shown that agricultural UAVs has unlimited potential in agriculture domain. However, there still several challenges and hurdles in these early stages of research and development with Artificial intelligence domain. In this work, we summarized the infrastructure, control, and applications of agricultural UAVs, which have been expanded or understudy. Besides, various issues, obtainable claims, and the latest results of agricultural UAVs are introduced to define the direction of future research work with AI.

\section{References}

C. W. Bac, E. J. van Henten, J. Hemming, and Y. Edan, "Harvesting robots for highvalue crops: State-of-the-art review and challenges ahead," Journal of Field
Robotics, vol. 31, no. 6, pp. 888-911, 2014

Klumpp, W. Ansel, G. Klumpp, P. Vergne, N. Sifakis, M. J. Sanz, S. Rasmussen, H. Ro-Poulsen, A` . Ribas, J. Peñuelas et al., "Ozone pollution and ozone biomonitoring in european cities part ii. ozoneinduced plant injury and its relationship with descriptors of ozone pollution," Atmospheric Environment, vol. 40, no. 38, pp. 7437-7448, 2006.

M. C. Hunter, R. G. Smith, M. E. Schipanski, L. W. Atwood, and D. A. Mortensen, "Agriculture in 2050: Recalibrating targets for sustainable intensication," Bioscience, vol. 67, no. 4, pp. 386-391, Feb. 2017.

N. Muchiri and S. Kimathi, "A review of applications and potential applications of UAV," in Proc. Sustain. Res. Innov. Conf., Jun. 2016, pp. 280-283.

O. Erat,W. A. Isop, D. Kalkofen, and D. Schmalstieg, "Drone-augmented human vision: Exocentric control for drones exploring hidden areas," IEEE Trans. Vis. Comput. Graphics, vol. 24, no. 4, pp. 1437-1446, Apr. 2018.

P. E. I. Pounds, D. R. Bersak, and A. M. Dollar, "Stability of small-scale UAV helicopters and quadrotors with added payload mass under PID control," Auton. Robots, vol. 33, nos. 1-2, pp. 129-142, 2012. 
S. Ward, J. Hensler, B. Alsalam, and L. F. Gonzalez, “Autonomous UAVs wildlife detection using thermal imaging, predictive navigation and computer vision," in Proc. IEEE Aerosp. Conf., Mar. 2016, pp. 1-8.

Sunny Thukral, Vijay Rana, (2018), Versatility of Fuzzy Logic in Chronic Diseases: A Review, Journal of Medical Hypotheses, Elsevier, Vol 122, pp 150-156.

T. C. Mallick, M. A. I. Bhuyan, and M. S. Munna, "Design \& implementation of an UAV (Drone) with ight data record," in Proc. Int. Conf. Innov. Sci., Eng.
Technol. (ICISET), Oct. 2016, pp. 1-6. Vijay Rana, Singh G, (2014) "An Analysis of Semantic Heterogeneity Issues and their Countermeasures Prevailing in Semantic Web", ICROIT 2014, IEEE Xplore, pp 16-22.

Vijay Rana, Singh G, (2014) "Analysis of Web Mining Technology and Their Impact on Semantic Web", CIPECH 2014, IEEE Xplore, pp 5-11.

W. J. Kim and J.-H. Kang, 'ToA-based localization algorithm for mitigating positioning error in NLoS channel," J. Inst. Control, Robot. Syst., vol. 24, no. 11, pp. 10431047, 2018.

\section{How to cite this article:}

Vijay Rana1 and Mahima. 2020. Impact of Drone Technology in Agriculture. Int.J.Curr.Microbiol.App.Sci. 9(01): 1613-1619. doi: https://doi.org/10.20546/ijcmas.2020.901.177 